\title{
Effectiveness of Fingolimod versus Natalizumab as Second-Line Therapy for Relapsing-Remitting Multiple Sclerosis in Spain: Second-Line GATE Study
}

\author{
José Meca-Lallana ${ }^{a}$ b Teresa Ayuso $^{c}$ Sergio Martínez-Yelamos ${ }^{d}$ Carmen Durán ${ }^{\text {e }}$ \\ Yessica Contreras Martín ${ }^{f}$ Nicolás Herrera Navarro ${ }^{g}$ Angel Pérez Sempere ${ }^{h}$ \\ Jose C. Álvarez-Cermeño ${ }^{\mathrm{i}}$ Jorge Millán Pascualj Virginia Meca-Lallanak \\ Raúl Romero Sevilla' Javier Ricart ${ }^{\prime}$

\begin{abstract}
aUnidad de Esclerosis Múltiple, Servicio de Neurología, Hospital Clínico Universitario "Virgen de la Arrixaca" (IMIBARRIXACA), Murcia, Spain; ${ }^{b}$ Cátedra de Neuroinmunología Clínica y Esclerosis Múltiple, UCAM, Universidad Católica

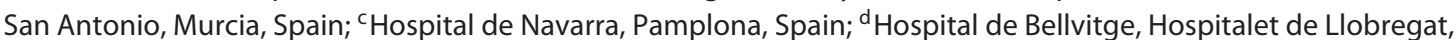

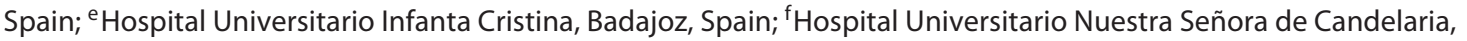

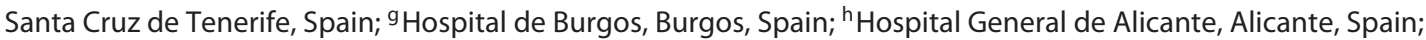
'Hospital Universitario Ramón y Cajal, IRYCIS, Madrid, Spain; 'Complejo Hospitalario La Mancha Centro, Alcázar de San Juan, Spain; ' Unidad de Enfermedades Desmielinizantes, Hospital Universitario de la Princesa, Madrid, Spain; 'Novartis Farmacéutica S.A., Barcelona, Spain
\end{abstract}

\section{Keywords}

Multiple sclerosis · Second-line treatment · Effectiveness · Fingolimod $\cdot$ Natalizumab

\begin{abstract}
Background: There is a lack of head-to-head studies comparing the efficacy of fingolimod (FIN) and natalizumab (NTZ) as second-line therapy for relapsing-remitting multiple sclerosis (RRMS). Methods: Multicenter, observational study, in which, information of 388 patients randomly selected and treated with FIN or NTZ in routine clinical practice was retrospectively collected with the main objective of comparing the annualized relapse rate (ARR) over the first year, after FIN or NTZ treatment initiation. Results: Mean ARR during the first year of treatment was 0.28 in FIN group and 0.12 in NTZ group ( $p=0.0064)$; nevertheless, the difference between groups lost statistical significance when the pro-
\end{abstract}

\begin{tabular}{ll}
\hline karger@karger.com & (c) 2020 The Author(s) \\
www.karger.com/ene & Published by S. Karger AG, Basel Openger \\
& This article is licensed under the Creative Commons Attribution- \\
Karger & NonCommercial-NoDerivatives 4.0 International License (CC BY- \\
NC-ND) (http://www.karger.com/Services/OpenAccessLicense). \\
Usage and distribution for commercial purposes as well as any dis- \\
tribution of modified material requires written permission.
\end{tabular}

pensity score analysis was performed. Time to disability progression was similar in both treatment groups (12.3 \pm 6.7 months in FIN, and $12.8 \pm 0.1$ months in NTZ; $p=0.4654$ ). Treatment persistence after the first year of treatment was higher in patients treated with FIN (95\%) than in those treated with NTZ (84\%; $p=0.0014)$. Conclusions: After 12 months of treatment, both FIN and NTZ reduced the ARR, but ARR percent reduction was significantly higher with NTZ. Treatment persistence was higher in patients receiving FIN.

$$
\begin{aligned}
& \text { C } 2020 \text { The Author(s) } \\
& \text { Published by S. Karger AG, Basel }
\end{aligned}
$$

\section{Introduction}

Multiple sclerosis (MS) is a chronic, inflammatory, demyelinating, and neurodegenerative disease that affects the central nervous system. The cause of MS is unknown, but it is thought to be an immune-mediated dis- 
ease $[1,2]$. Relapsing-remitting MS (RRMS) is characterized by clearly defined relapses of new or increasing neurologic symptoms. These relapses are followed by periods of partial or complete recovery (remissions) [3, 4]. Most patients start with a relapsing-remitting course, which may be followed by a secondary progressive phase. A minority of subjects present with an insidious progression of disability from onset, or primary progressive [5]. Current first-line disease-modifying therapies (DMT), interferon beta and glatiramer acetate, are partially effective for the treatment of RRMS. Treatment strategies for patients with suboptimal response to a first-line DMT include the escalation to a more efficacious DMT, fingolimod (FIN; a sphingosine 1-phopahte receptor modulator), or natalizumab (NTZ; an a4integrin monoclonal antibody) [6]. Until present, there are no head-to-head randomized clinical trials that have compared the clinical efficacy of FIN and NTZ in patients with RRMS nonresponding to first-line DMT; nevertheless, NTZ is perceived as more efficacious drug than FIN, perception that has not been proved neither in clinical research nor in clinical practice. Factors that may have contributed to this perception are (i) NTZ was first marketed and was used as the only second-line DMT for several years and (ii) the subsequent marketing authorization of FIN relegated this drug to the treatment of patients that failed a previous therapy with NTZ or had an increased risk of developing progressive multifocal leukoencephalopathy. In addition, MS relapses have been observed in $30-40 \%$ of the patients after switching from NTZ to FIN [7-9].

The objective of this study was to compare the effectiveness of FIN versus NTZ administered in routine clinical practice as a second-line therapy, in patients with RRMS.

\section{Materials and Methods}

This was a national, multicenter, retrospective with randomized patient selection, observational study carried out in Spain. The information of patients aged 18-55 years, with MS diagnosed according to $2010 \mathrm{McDonald}$ criteria [10], a relapsing-remitting course [4], and treated with FIN or NTZ between November 2011 and April 2013 with, at least, 12 months of follow-up, was retrospectively collected from their medical records at 33 hospital outpatient Neurology Services with medical activity dedicated to patients with RRMS. FIN and NTZ were prescribed in accordance with the local marketing authorization. Exclusion criteria included a clinical course different to RRMS; prior treatment with FIN, NTZ, immunoglobulins, cyclophosphamide, cladribine, mitoxantrone, and/or monoclonal antibodies at any moment; and treatment with immunosuppressants or investigational DMT within the 12 months prior to FIN or NTZ initiation. Naïve patients whose first DMT was FIN or NTZ for rapidly evolving severe RRMS were also excluded [11]. To minimize potential selection bias, patients that fulfilled all eligibility criteria were randomly selected in each center.

The study was conducted in accordance with ethical principles that have their origin in the Declaration of Helsinki. The study was approved by the Spanish Agency of Medicines and Medical Devices, and the Ethics Committees of Hospital Infanta Cristina, Hospital de Bellvitge, Hospital de Navarra, and Hospital Virgen de la Arrixaca.

The primary endpoint of the study was the difference between treatments in the annualized relapse rate (ARR) over the first year, after FIN or NTZ treatment initiation. Secondary endpoints included the number of relapses, time to first relapse, proportion of relapse-free patients, proportion of patients with confirmed disability progression (increase of $\geq 1.5$ points on the Expanded Disability Status Scale (EDSS) score from a baseline score of $<1$, or increase $\geq 1$ point from a baseline score $\geq 1$, confirmed after, at least, 3 months), number of T1 Gd-enhancing lesions, proportion of patients free of T1 Gd-enhancing lesions, proportion of patients free of new or enlarging T2-hyperintense lesions, proportion of patients free of radiologic activity (defined as the absence of T1 Gd-enhancing lesions and new or increased T2 lesions), proportion of patients free of clinical activity (defined as the absence of relapses and sustained disability progression), proportion of patients free of disease (defined as the absence of clinical and radiologic activity), number of adverse events (AE) during the observation of the first dose administered, number of AEs after treatment initiation, and proportion of patients that interrupted the study within the first year after 2nd-line treatment initiation.

\section{Statistical Analysis}

The primary endpoint of the study was the ARR over the first year, after FIN or NTZ treatment initiation. An analysis of covariance was used to assess the percent change on the ARR adjusting for the EDSS score as it was the only variable that showed a significant difference at baseline. In addition, to define comparable groups to assess the ARR, a propensity score (PS) analysis matching 1:1 was performed. Patients were PS matched on age, EDSS score, number of T1 Gd-enhancing lesions, and number of T2 lesions when starting on 2nd-line therapy, ARR (over the previous year), and time passed since RRMS diagnosis.

Continuous variables are presented as means, standard deviations, and 95\% CI, as appropriate. Categorical variables are reported by using frequencies and percentages.

Descriptive statistics were calculated for demographic and clinical characteristics.

The number of relapses was analyzed with the Mann-Whitney $\mathrm{U}$ test. Kaplan-Meier was used to assess the time to first relapse since treatment initiation and the time to disability progression. Comparisons between groups regarding the percentage of patients free of clinical activity, disease, or radiologic activity, and the percentage of patients that discontinued the study were assessed using the chi-square or Fisher exact test. Comparison between groups regarding the number of lesions observed in MRI was assessed using the Wilcoxon signed-rank test.

A 2 -tailed $p$ value $<0.05$ was used to denote statistically significant differences.

All statistical analyses were performed with $\mathrm{SAS}^{\circledR}$ software version 9.4 (SAS Institute Inc., Cary, NC, USA).
Eur Neurol 2020;83:25-33 DOI: $10.1159 / 000505778$
Meca-Lallana et al. 
Table 1. Clinic-demographic characteristics of the patients at baseline (2nd-line treatment initiation)

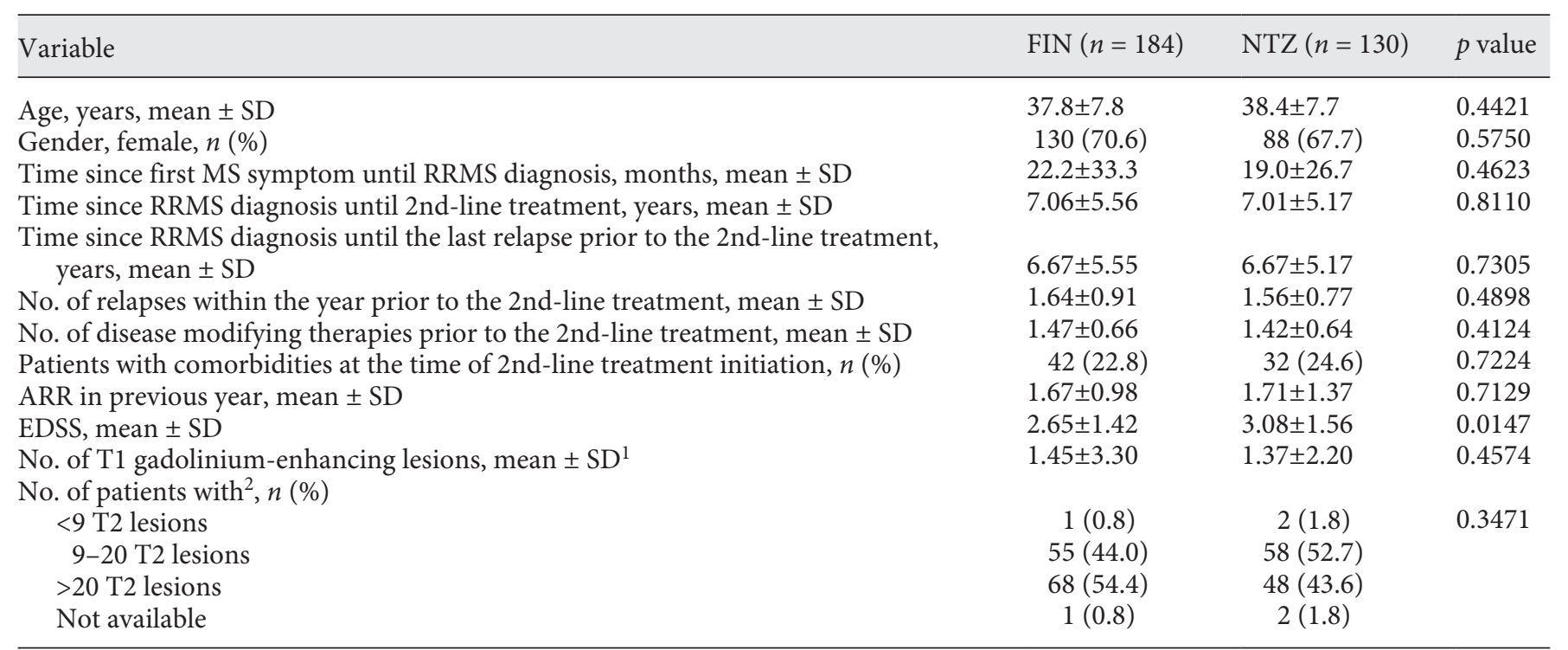

${ }^{1}$ Only in patients with MRI available. For this variable, no. of evaluable patients $=121$ for FIN group and $n=106$ for NTZ group.

${ }^{2}$ Only in patients with MRI available. For this variable, no. of evaluable patients $=125$ for FIN group and $n=110$ for NTZ group.

FIN, fingolimod; NTZ, natalizumab; SD, standard deviation; MS, multiple sclerosis; RRMS, relapsing-remitting MS; ARR, annualized relapse rate; EDSS, Expanded Disability Status Scale.

\section{Results}

The study included 388 patients; of them, 74 (19.1\%) were excluded from the analysis, $33(8.5 \%)$ due to lack of information regarding the primary endpoint (AAR), 32 (8.2\%) for not fulfilling the eligibility criteria, and 9 (2.4\%) for other reasons. Thus, 314 (80.9\%) patients were evaluable. A reduced population of 260 patients ( 130 patients per group) was obtained after the PS matching 1:1 used for the additional analysis of the ARR.

Out of the 314 evaluable patients, 184 (58.6\%) patients were treated with FIN and 130 (41.4\%) with NTZ. Clinical and demographic characteristics of both groups at the 2nd-line treatment initiation were similar, only a statistically significant difference was found in the mean EDSS score $(2.65 \pm 1.42$ in FIN group versus $3.08 \pm 1.56$ in NTZ group, $p=0.0147$; Table 1 ).

Considering the whole study population, and compared with the previous year, during the first year of treatment, the ARR showed a statistically significant reduction in both patient groups, from $1.67 \pm 0.98$ to $0.28 \pm 0.62$ in FIN group $(p<0.0001)$ and from $1.71 \pm 1.37$ to $0.12 \pm 0.33$ in NTZ group $(p<0.0001)$. Nevertheless, the ARR during the first year of treatment with the study drugs was sig- nificantly lower in NTZ group ( $p=0.0064)$. Differences between groups in the ARR mean percent change were only statistically significant during the first year of treatment (Fig. 1). Very similar results were found when the PS was used to, additionally, analyze the ARR. Nevertheless, with the PS analysis, the difference between groups in the ARR mean percent change observed in the first year lost the statistical significance $(-83.5 \%$ [ $95 \%$ CI -90.2 to -76.7 ] in FIN group versus $-92.3 \%$ [95\% CI -96.2 to -88.3 ] in NTZ group, [ $p=0.0958]$ ).

The mean number of relapses during the first year of treatment was higher in FIN group $(0.28 \pm 0.62)$ than in the group of patients treated with NTZ $(0.12 \pm 0.33 ; p=$ $0.0237)$. After the first year, the mean number of relapses in both groups was similar $(0.15 \pm 0.42$ vs. $0.12 \pm 0.34$ during the 2nd year, in FIN group versus NTZ group, respectively $[p=0.5211]$; and $0.10 \pm 0.38$ vs. $0.10 \pm 0.33$ during the 3rd year in FIN vs. NTZ group, respectively [ $p=$ $0.8001])$.

The cumulative probability of remaining free of relapse was higher in NTZ group (61.5\% in FIN group and $71.5 \%$ in NTZ group, at 48 months [HR 1.56; 95\% CI $1.00-2.42$; $p=0.0450]$; Fig. 2). Considering only the patients that relapsed, no statistically significant differences 


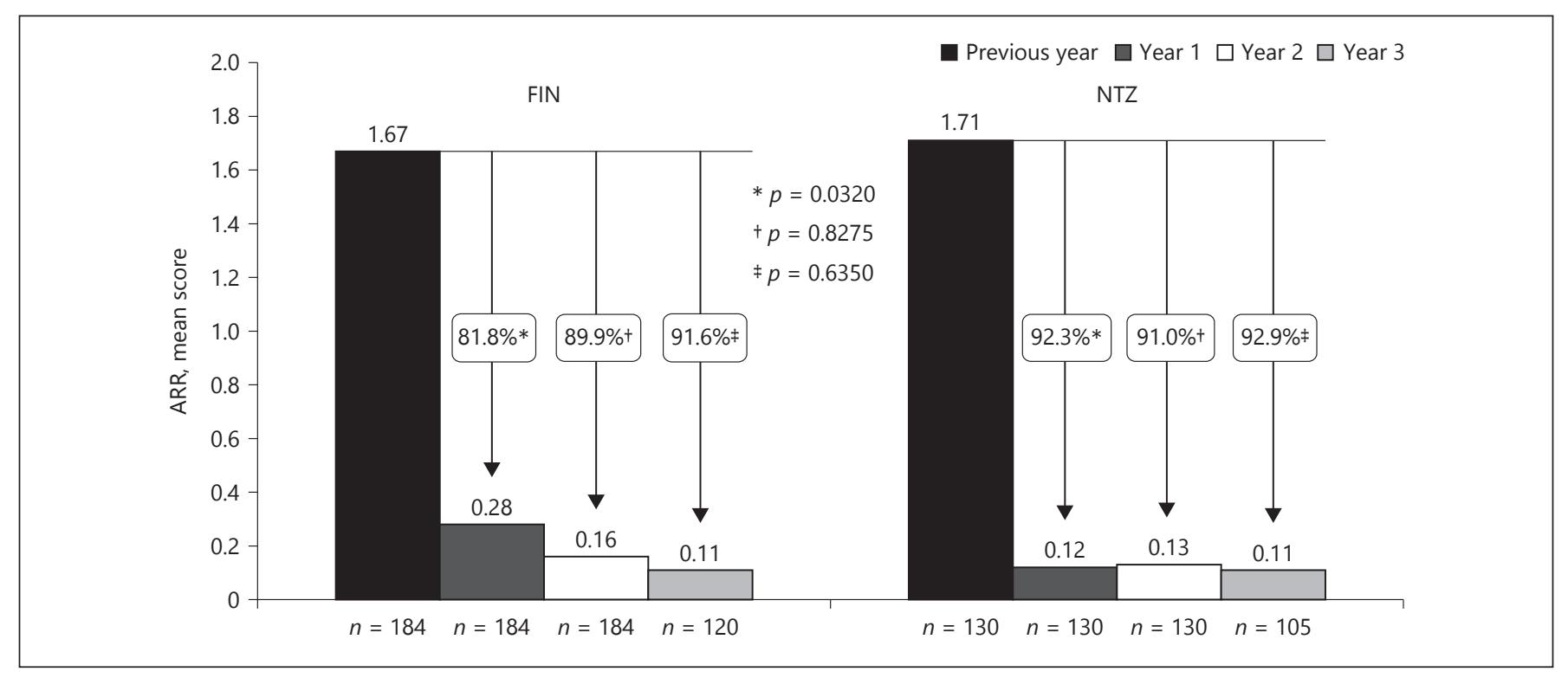

Fig. 1. ARR according to treatment group (total study population). ${ }^{*,+}{ }^{\ddagger} p$ value for percent change comparison between treatment groups regarding the year of follow-up (* year $1,{ }^{\dagger}$ year 2 , and ${ }^{\ddagger}$ year 3 ). FIN, fingolimod; NTZ, natalizumab; ARR, annualized relapse rate.

were found in the mean time to first relapse since 2 nd-line treatment initiation (10.8 months [95\% CI 8.6-13.1] in FIN group, and 13.5 months [95\% CI 9.7-17.4] in NTZ group $[p=0.3137])$.

Compared to baseline, 12 months after 2nd-line treatment initiation, the EDSS mean percent reduction was similar in both groups $(-8.86 \%$ [ $95 \%$ CI -14.01 to -3.71 ] and $-8.88 \%$ [ $95 \%$ CI -17.29 to -0.46 ] in FIN and NTZ group, respectively [ $p=0.1628]$ ).

No statistically significant differences were found in confirmed disability progression at month 12 after 2ndline treatment initiation $(6.5 \%$ of the patients in FIN group, and $4.6 \%$ in NTZ group; $p=0.7944$ ). The proportion of patients showing confirmed disability progression at month 24 was 12.6 and $8.1 \%$ in FIN group and NTZ group, respectively ( $p=0.3248$; Fig. 3 ). Mean time to disability progression was $12.3 \pm 6.7$ months in FIN group and $12.8 \pm 0.1$ months in NTZ group $(p=0.4654)$.

Table 2 shows the radiologic activity assessed in a subgroup of 95 patients ( 49 FIN and 46 NTZ) with MRI available at the beginning of the 2nd-line treatment initiation and annual MRIs throughout the study. Twelve months after 2nd-line treatment initiation, results from the analysis of the number of $\mathrm{T} 1$ gadolinium-enhancing lesions, the proportion of patients free of $\mathrm{T} 1 \mathrm{Gd}$-enhancing lesions, and the proportion of patients free of new or enlarging T2-hyperintense lesions favored NTZ. At month
24, differences between treatment groups lost statistical significance.

No statistically significant differences were found in the proportion of patients free of clinical activity after 12 months of treatment with the 2nd-line drugs (73.1\% in FIN group and $82.4 \%$ in NTZ group; $p=0.1140$ ). Considering patients with continued treatment and MRI valid for assessment at month $12(n=149)$, the proportion of patients free of radiologic activity was $63.5 \%$ in FIN group and $96.0 \%$ in NTZ group $(p<0.0001)$, and the proportion of patients free of disease was $50.0 \%$ in FIN group and $78.7 \%$ in NTZ group ( $p=0.0002)$.

Table 3 summarizes the AEs reported over the observation period for the first dose of the 2nd-line treatment and those reported afterward throughout the study. Infection was the most common AE in each group; nevertheless, its incidence was lower in FIN group (23.6\%) than in NTZ group (57.1\%; $p=0.0005)$.

During the first year on 2 nd-line therapy, $1.1 \%$ of the patients in FIN interrupted the treatment permanently and $5.4 \%$ temporarily; whereas in the NTZ group, $1.4 \%$ interrupted the treatment permanently and $2.3 \%$ temporarily. AEs were the most common reasons for temporary interruptions (in $5.4 \%$ of FIN patients and in $1.5 \%$ of NTZ patients). The most common reasons for permanent interruptions were lack of effectiveness and patient decision in FIN group (1.1\% for each reasons), and positive serology 


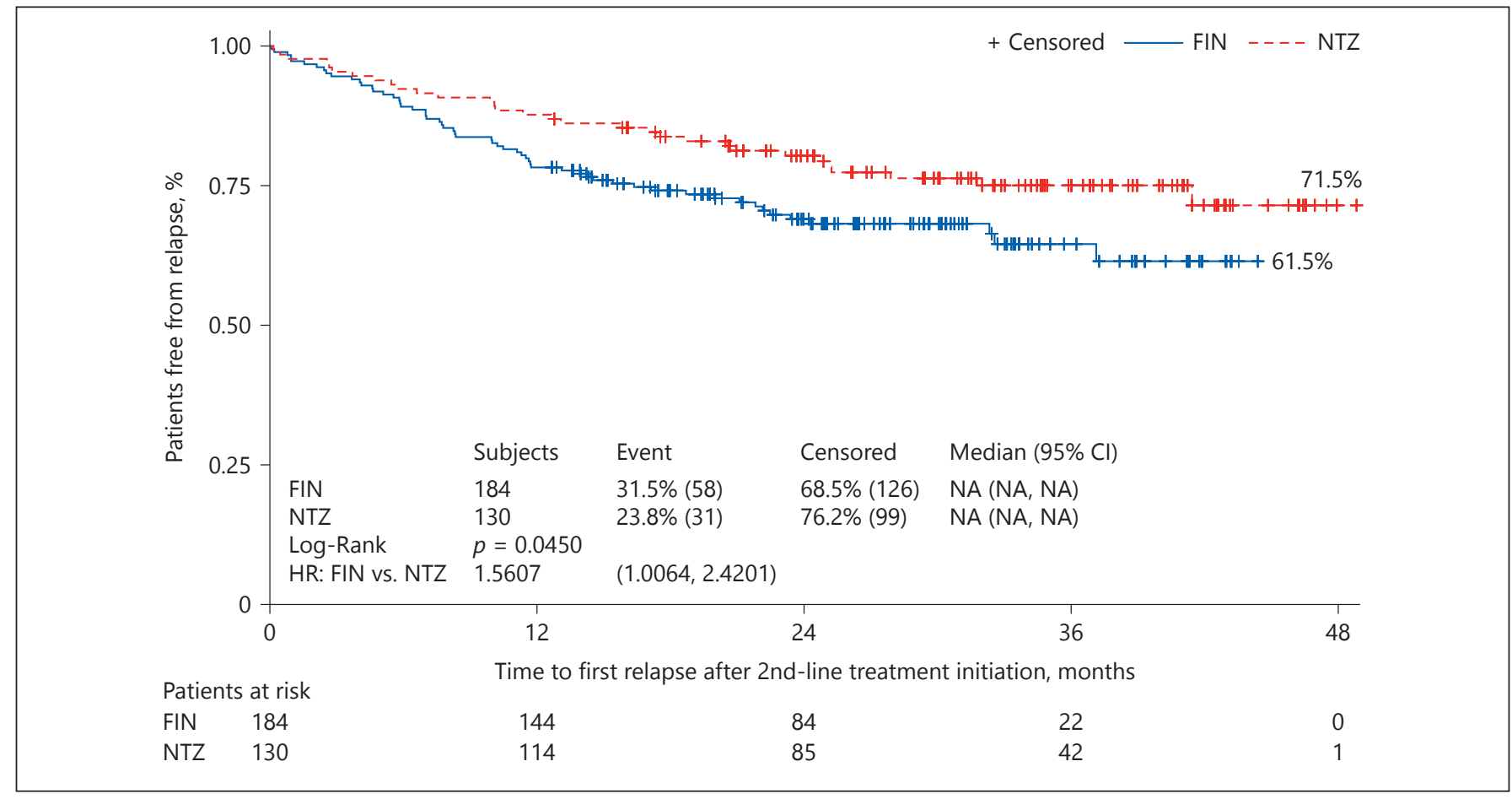

Fig. 2. Time to first relapse. FIN, fingolimod; NTZ, natalizumab; HR, hazard ratio.

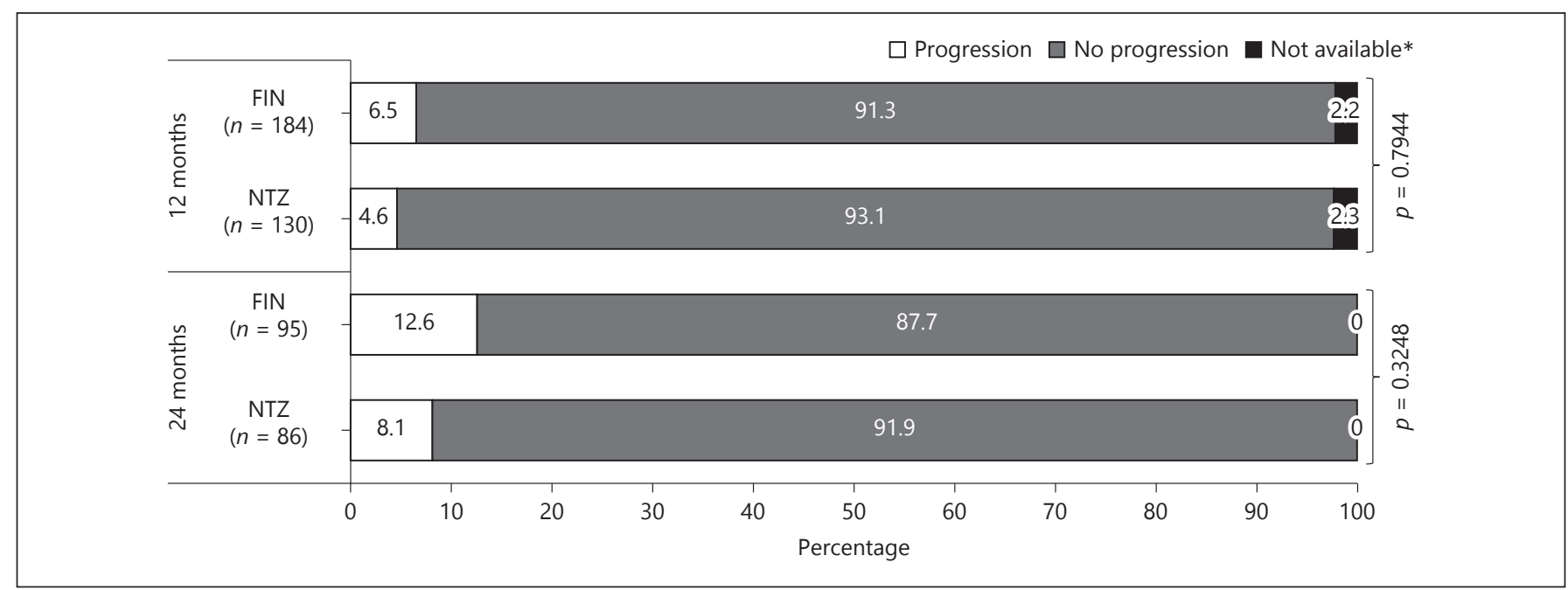

Fig. 3. Proportion of patients with confirmed disability progression after 12 and 24 months of 2 nd-line treatment initiation. Confirmed disability progression was defined as an increase of $\geq 1.5$ points on the EDSS score from a baseline score of $<1$, or an increase $\geq 1$ point from a baseline score $\geq 1$, confirmed after, at least, 3 months. As the EDSS score is assessed at 3, 6, and 12 months during the first year of follow-up, if disease progression was ob- served at month 3, confirmation was assessed at month 6; if disease progression was observed at month 6 , confirmation was assessed at month 12; and if disease progression was observed at month 12, confirmation was assessed at month $18 .{ }^{*}$ When disease progression was observed at month 12 , but EDSS score was not available afterwards to assess confirmation. FIN, fingolimod; NTZ, natalizumab. 
Table 2. MRI activity at baseline (2nd-line treatment initiation), 12 and 24 months, according treatment group

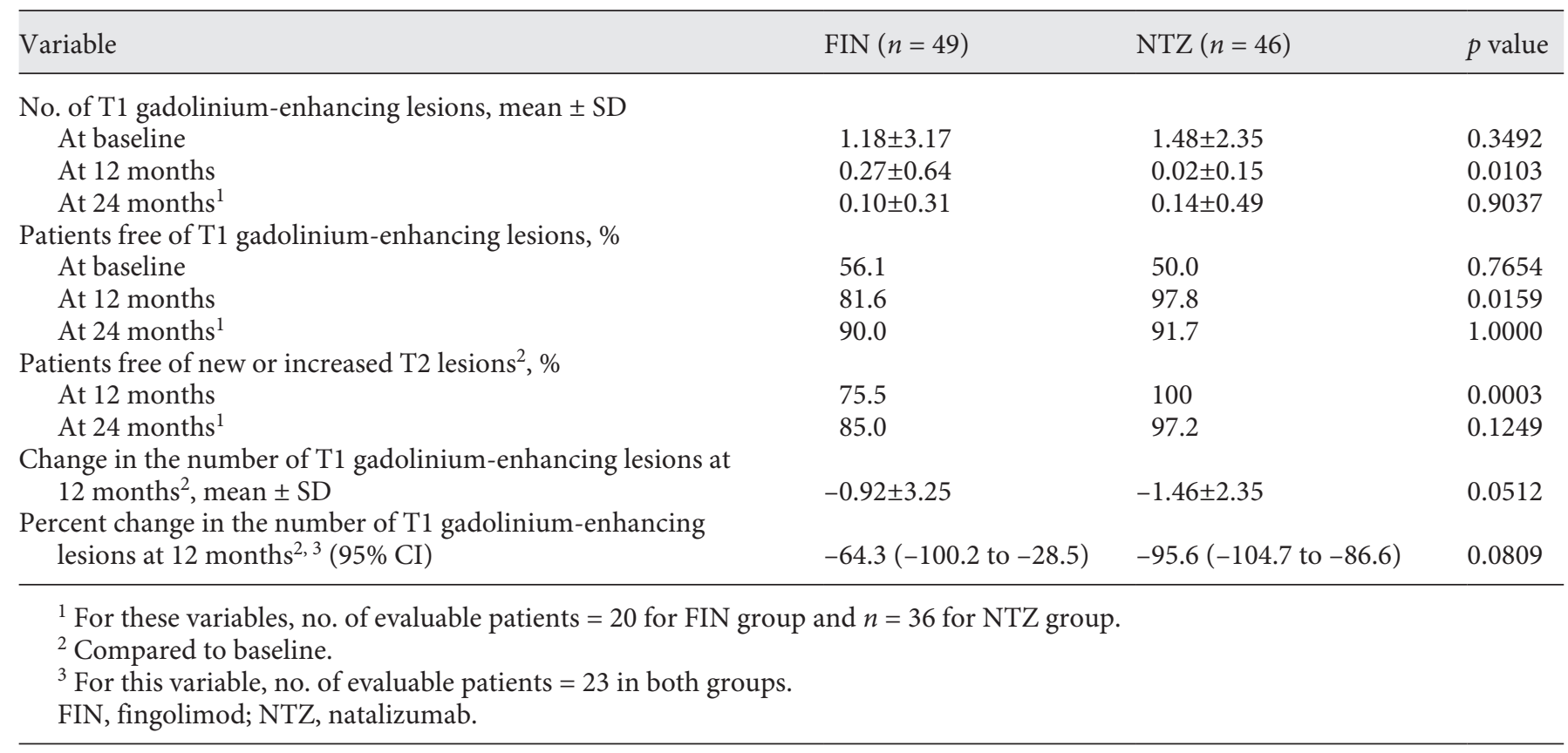

Table 3. Adverse events

FIN $(n=184), n(\%) \quad \mathrm{NTZ}(n=130), n(\%)$

AEs reported over the observation period for the first dose of the 2nd-line treatment ${ }^{1}$

Patients with, at least, one AE

Total number of $\mathrm{AEs}^{2}$

Infusion reaction

First-degree atrioventricular block

Other

AEs reported throughout the study, since 2nd-line treatment initiation ${ }^{3}$

Patients with, at least, one AE

Total number of $\mathrm{AEs}^{2,4}$

Infection

Increased liver enzymes

Lymphopenia $(<200$ lymphocytes $/ \mu \mathrm{L})$

Infusion reaction

Symptomatic bradycardia

Macular edema

Immune reconstitution inflammatory syndrome

Other

$\begin{array}{ll}2(1.1) & 5(3.8) \\ 2 & 6 \\ 0(0.0) & 2(33.3) \\ 1(50.0) & 0(0.0) \\ 1(50.0) & 4(66.7)\end{array}$

More than one AE could be reported by the same patient.

${ }^{1}$ AEs reported the same day or the day after the first dose administration (at the beginning of the treatment or once the treatment had been reintroduced after a temporal treatment interruption).

${ }^{2}$ Percentages calculated over the total number of AEs in each group.

${ }^{3}$ AEs reported over the observation period for the first dose have been excluded.

${ }^{4}$ Differences between groups not statistically significant, except for "Infection" ( $\left.p=0.0005\right)$ and "Other" $(p=0.0248)$.

AEs, adverse events; FIN, fingolimod; NTZ, natalizumab.

$\begin{array}{cc}41(22.8) & 20(15.4) \\ 55 & 49 \\ 13(23.6) & 28(57.1) \\ 7(12.7) & 2(4.1) \\ 3(5.4) & 0(0.0) \\ 0(0.0) & 2(4.1) \\ 0(0.0) & 1(2.0) \\ 2(3.6) & 0(0.0) \\ 1(1.8) & 0(0.0) \\ 30(54.5) & 16(32.6)\end{array}$


for the John Cunningham virus in NTZ group (1.5\%). Mean time of treatmentinterruption was $1.82 \pm 0.90$ months in FIN group and $2.60 \pm 1.35$ months in NTZ group.

Treatment persistence after the first year was higher in patients treated with FIN (94.6 vs. $83.9 \%$ in NTZ; $p=$ $0.0014)$.

\section{Discussion/Conclusion}

In this retrospective, observational study, we compared the effectiveness of FIN and NTZ in RRMS patients failing on a first-line therapy in clinical practice in Spain. The clinical and demographic characteristics of the patients were similar in both treatment groups at the time of 2nd-line therapy initiation; only EDSS score was significantly higher in NTZ group. Over the first year of treatment, and compared with baseline, both FIN and NTZ reduced significantly the ARR. Considering the whole study population, we found a significant higher percent reduction in patients treated with NTZ during the first year, but differences lost the statistical significance at the 2nd and 3rd year. As EDSS score was higher in NTZ at the beginning of the 2nd-line therapy, and treatment response can be influenced by the disease activity [12], the 2 patient groups analyzed could have not been comparable. To avoid this potential bias, the PS was used to analyze the ARR and, as a result, no statistically significant differences were found at any time-point. In any case, this finding should be considered with caution, as the loss of the statistical significance may be attributable to the lower number of patients included in the analysis after being matched. Reviewing other noninterventional studies, we found contradictory results; some studies [13-15] showed higher reduction in ARR with NTZ when compared to FIN, but others showed no differences between both drugs $[16,17]$. In line with our results, in 2 $[13,15]$ of the 3 studies where differences favored NTZ analyzing the whole study population, these differences disappeared when PS was applied.

As already observed in 4 noninterventional studies $[14,18-20]$, in our study, after 2 years of treatment, the probability of remaining free of relapse was higher in NTZ group than in FIN group. In contrast, no significant differences between treatment groups in the proportion of patients free of relapses have been observed in another 5 noninterventional studies [13, 15-17, 21].

We found differences favoring NTZ in the EDSS scores at month 12. This is in line with the study of Kalincik et al. [14] in which a higher proportion of patients treated with NTZ, compared with those treated with FIN, experienced 6-month sustained regression disability. Lanzillo et al. [15] also found a change in EDSS after 2 years of therapy favoring NTZ, but statistical significance disappeared when adjusting for the PS. On the contrary, Baroncini et al. [18] found, in unmatched population, a higher risk for EDSS worsening in patients treated with NTZ but, when adjusting for PS, a trend favoring NTZ was found. In other studies $[13,16,17,20,22]$, no differences were found between FIN and NTZ in the evolution of the EDSS score.

After 1 and 2 years of follow-up, 3 studies found a lower proportion of MRI-active patients in NTZ, when compared to FIN [18, 19, 22]. Conversely, Gajoffato et al. [22] did not find differences between the 2 drugs on MRI outcomes after a mean of 13 months of follow-up. In our study, higher improvements in MRI outcomes were seen at month 12 in NTZ. Nevertheless, differences at 24 months were not statistically significant.

Both, FIN and NTZ, were well tolerated, and the AEs reported by the participants in our study are consistent with the safety information from randomized clinical trials $[23,24]$. Notably, the incidence of infections was higher in NTZ. Few patients from both treatment groups discontinued the study due to AEs; nevertheless, persistence after the first year was higher in patients that were started on FIN than in those that started on NTZ. Probably this was a consequence of NTZ discontinuation due to safety concerns as the risk of progressive multifocal leukoencephalopathy associated with the use of NTZ is well known $[25,26]$. In line with our results, higher rates of discontinuations in patients receiving NTZ have also seen by Gajofatto et al. [22] and Guger et al. [13]. Contrarily, Frisell et al. [27] found higher proportion of patients remaining on NTZ than on FIN but differences in discontinuation rates between FIN y NTZ disappeared when a covariate-adjusted analysis was applied.

Focusing on the group of patients treated with FIN, the results obtained in our study were very similar to those found in a large cohort of Spanish patients $(n=211)$ in which the effectiveness and safety of FIN for the treatment of RRMS were also retrospectively assessed [28]. In that cohort, the ARR after 24 months of treatment with FIN was 0.3 , compared to an ARR of 0.16 observed in our study after 24 months of treatment. The proportion of relapse-free patients at month 36 was $62 \%$, compared with $61.5 \%$ at month 48 in our study, and the percentage of patients with confirmed disability progression at month 12 was $6.9 \%$, compared to $6.5 \%$ in our study. Differently from our study population, $20.4 \%$ of the patients 
in that cohort were previously treated with NTZ, and $20.8 \%$ were treatment-naïve patients. Despite the differences between the 2 populations, the similarity of the results obtained gives us a clear idea of what FIN, administered in real-life clinical practice, can offer.

As in other observational studies, our main limitation was the potential patient selection bias. To minimize this bias, patients were randomly selected from those that fulfilled the inclusion and exclusion criteria in each of the participant sites. Another potential source of bias is the selection of the treatment that, in routine clinical practice, can be influenced by the patient's profile and/or the physician's preference. Despite this, we found that only the EDSS score differed significantly at baseline. When PS matching 1:1 was used to control confounding covariates that could have an effect in the analysis of our main endpoint (ARR), the number of patients was reduced and, in consequence, the statistical power for this analysis. Another limitation was that MRI data at 24 months were only available in a small number of patients; therefore, the results observed when the MRI outcomes were analyzed at that time point should be considered with caution.

The strengths of our study are that this was a multicenter, observational study with randomized selection of patients at each site. As this was a real-life study, the results can be considered meaningful to everyday clinical practice.

In conclusion, after 12 months of treatment both FIN and NTZ reduced the ARR, but ARR percent reduction was significantly higher with NTZ although these differences were minimized, and the statistical significance was lost in the second and third year. More beneficial effect on radiologic activity was also obtained with NTZ during the first year of treatment. Treatment persistence was significantly higher in patients receiving FIN.

Head-to-head clinical studies comparing FIN versus NTZ are warranted to clarify the contradictory results found in published observational studies.

\section{Acknowledgments}

We thank Veronica Alfonso at TFS Trial Form Support for statistical analysis assistance, and Angel Burgos at PIVOTAL for editing the manuscript and editorial assistance. We also thank the investigators participating in the study: Y. Aladro (H. de Getafe), S. Moreno García (H. 12 de Octubre); J. Gracia Gil, E. Fernández Díaz (H. General Universitario de Albacete); J.M. García Domínguez (H. Gómez-Ulla); C. López de Silanes de Miguel (H. Universitario de Torrejón); J. Olascoaga Utaza, I. Lopetegui Larruscain, I. Croitoru (H. Universitario de Donostia); I. García Castañón (H. Universitario de Fuenlabrada); F. Coret Ferrer, F. Gascón
Giménez, (H. Clínico Universitario de Valencia); J. Mallada Frechín (H. General Universitario de Elda); M. Carcelén Gadea, L.F. Gómez Betancur (H. General Universitario de Valencia); C. Calles Hernández, M. Massot Cladera (H. Universitario Son Espases); A. Escartín Siquier, M. López Góngora, S. Figueroa Bonaparte (H. de la Santa Creu i Sant Pau); M. Gómez Gutiérrez, R. Romero Sevilla (H. San Pedro de Alcántara); A. Oterino, V. González Quintanilla (H. Marqués de Valdecilla); E. Costa Arpin, J.M. Prieto González (H. Clínico Universitario de Santiago); D. Muñoz García, E. Álvarez Rodríguez, C. Valerias Muñoz (H. Xeral de Cíes); Y. Blanco Morgado (H. Clínic de Barcelona); X. Montalbán, B. Rodríguez, J. Castillo, M. Tintoré, A. Vidal, J. Sastre, C. Nos (H. Universitari Vall d'Hebrón); C. Muñoz Fernández (Complejo Hospitalario Torrecárdenas); R. Fernández-Bolaños (H. Universitario de Valme); P. Oliva Nacarino, J. Villafani Echazu (H. Central de Asturias); A. Ares Luque, L. Redondo Robles (H. de León); G. Navarro Mascarell, J.L. Ruiz Peña (H. Virgen Macarena); M.A. Hernández Pérez, Y. Marrero Curbelo, Y. Contreras Martín (H. Universitario Nuestra Señora de Candelaria); V. Galán Sánchez-Seco (H. Clínico San Carlos); J. Millán Pascual (Complejo Hospitalario La Mancha Centro); V. Meca-Lallana (H. Universitario de La Princesa); A. Pérez Sempere (H. General Universitario de Alicante); N. Herrera Navarro, H. Monteiro Matos, B. García (H. Universitario de Burgos); L. Costa-Frossard Franca, J.C. Álvarez-Cermeño. (H. Ramón y Cajal), and R. Hernández Clares, E. Carreón Guarnizo, M. Cerdán Sánchez (H. Universitario Virgen de la Arrixaca).

\section{Statement of Ethics}

The study was conducted in accordance with ethical principles that have their origin in the Declaration of Helsinki. The study was approved by the Spanish Agency of Medicines and Medical Devices and the Ethics Committees of Hospital Infanta Cristina, Hospital de Bellvitge, Hospital de Navarra, and Hospital Virgen de la Arrixaca.

\section{Disclosure Statement}

The authors declare that they have no conflicts of interest.

\section{Funding Sources}

This study was funding by Novartis Farmacéutica, S.A.

\section{Author Contributions}

Drs. José Meca-Lallana, Teresa Ayuso, Sergio Martinez-Yelamos, Carmen Durán, Yessica Contreras Martín, Nicolás Herrera Navarro, Angel Perez Sempere, Jose C. Álvarez-Cermeño, Jorge Millán Pascual, and Virginia Meca-Lallana: research project execution and manuscript review and critique. Drs. Raúl Romero Sevilla and Javier Ricart: research project design and statistical analysis review and critique and manuscript review and critique. 


\section{References}

1 Compston A, Coles A. Multiple sclerosis. Lancet. 2008 Oct;372(9648):1502-17.

2 Loma I, Heyman R. Multiple sclerosis: pathogenesis and treatment. Curr Neuropharmacol. 2011 Sep;9(3):409-16.

3 Lublin FD, Reingold SC; National Multiple Sclerosis Society (USA) Advisory Committee on Clinical Trials of New Agents in Multiple Sclerosis. Defining the clinical course of multiple sclerosis: results of an international survey. Neurology. 1996 Apr;46(4):907-11.

4 Lublin FD, Reingold SC, Cohen JA, Cutter GR, Sørensen PS, Thompson AJ, et al. Defining the clinical course of multiple sclerosis: the 2013 revisions. Neurology. 2014 Jul;83(3): 278-86.

5 Reynders T, D'haeseleer M, De Keyser J, Nagels G, D’hooghe MB. Definition, prevalence and predictive factors of benign multiple sclerosis. eNeurologicalSci. 2017 May;7:37-43.

6 Freedman MS. Treatment options for patients with multiple sclerosis who have a suboptimal response to interferon-beta therapy. Eur J Neurol. 2014;21:377-87.e18-20.

7 Rinaldi F, Seppi D, Calabrese M, Perini P, Gallo P. Switching therapy from natalizumab to fingolimod in relapsing-remitting multiple sclerosis: clinical and magnetic resonance imaging findings. Mult Scler. 2012 Nov; 18(11) 1640-3.

8 Sempere AP, Martín-Medina P, BerenguerRuiz L, Pérez-Carmona N, Sánchez-Perez R, Polache-Vengud J, et al. Switching from natalizumab to fingolimod: an observational study. Acta Neurol Scand. 2013 Aug;128(2): e6-10.

9 Cohen M, Maillart E, Tourbah A, De Sèze J, Vukusic S, Brassat D, et al.; Club Francophone de la Sclérose en Plaques Investigators. Switching from natalizumab to fingolimod in multiple sclerosis: a French prospective study. JAMA Neurol. 2014 Apr;71(4):436-41.

10 Polman CH, Reingold SC, Banwell B, Clanet M, Cohen JA, Filippi M, et al. Diagnostic criteria for multiple sclerosis: 2010 revisions to the McDonald criteria. Ann Neurol. 2011 Feb; 69(2):292-302.

11 Devonshire V, Havrdova E, Radue EW, O'Connor P, Zhang-Auberson L, Agoropoulou $\mathrm{C}$, et al.; FREEDOMS study group. Re- lapse and disability outcomes in patients with multiple sclerosis treated with fingolimod: subgroup analyses of the double-blind, randomised, placebo-controlled FREEDOMS study. Lancet Neurol. 2012 May;11(5):420-8.

12 Peño L, Seco V, Valencia C, García-Montojo M, Domínguez-Mozo MI, García-Martínez MA, et al. Clinical data associated with the therapeutic response to glatiramer acetate in multiple sclerosis patients. Neuro Open J. 2016;3(1):3-8.

13 Guger M, Enzinger C, Leutmezer F, Kraus J, Kalcher S, Kvas E, et al. Real-life clinical use of natalizumab and fingolimod in Austria. Acta Neurol Scand. 2018 Feb;137(2):181-7.

14 Kalincik T, Horakova D, Spelman T, Jokubaitis $\mathrm{V}$, Trojano M, Lugaresi A, et al.; MSBase Study Group. Switch to natalizumab versus fingolimod in active relapsing-remitting multiple sclerosis. Ann Neurol. 2015 Mar;77(3): 425-35.

15 Lanzillo R, Carotenuto A, Moccia M, Saccà F, Russo CV, Massarelli M, et al. A longitudinal real-life comparison study of natalizumab and fingolimod. Acta Neurol Scand. 2017 Sep; 136(3):217-22.

16 Braune S, Lang M, Bergmann A; NTC Study Group. Second line use of Fingolimod is as effective as Natalizumab in a German out-patient RRMS-cohort. J Neurol. 2013 Dec;260(12): 2981-5.

17 Koch-Henriksen N, Magyari M, Sellebjerg F, Soelberg Sørensen P. A comparison of multiple sclerosis clinical disease activity between patients treated with natalizumab and fingolimod. Mult Scler. 2017 Feb;23(2):234-41.

18 Baroncini D, Ghezzi A, Annovazzi PO, Colombo B, Martinelli V, Minonzio G, et al. Natalizumab versus fingolimod in patients with relapsing-remitting multiple sclerosis nonresponding to first-line injectable therapies. Mult Scler. 2016 Sep;22(10):1315-26.

19 Barbin L, Rousseau C, Jousset N, Casey R, Debouverie M, Vukusic S, et al.; CFSEP and OFSEP groups. Comparative efficacy of fingolimod vs natalizumab: A French multicenter observational study. Neurology. 2016 Feb; 86(8):771-8.

20 Prosperini L, Saccà F, Cordioli C, Cortese A, Buttari F, Pontecorvo S, et al. Real-world ef- fectiveness of natalizumab and fingolimod compared with self-injectable drugs in nonresponders and in treatment-naïve patients with multiple sclerosis. J Neurol. 2017 Feb; 264(2):284-94.

21 Carruthers RL, Rotstein DL, Healy BC, Chitnis T, Weiner HL, Buckle GJ. An observational comparison of natalizumab vs. fingolimod using JCV serology to determine therapy. Mult Scler. 2014 Sep;20(10):1381-90.

22 Gajofatto A, Bianchi MR, Deotto L, Benedetti $\mathrm{MD}$. Are natalizumab and fingolimod analogous second-line options for the treatment of relapsing-remitting multiple sclerosis? A clinical practice observational study. Eur Neurol. 2014;72(3-4):173-80.

23 Kappos L, Radue EW, O'Connor P, Polman C, Hohlfeld R, Calabresi P, et al.; FREEDOMS Study Group. A placebo-controlled trial of oral fingolimod in relapsing multiple sclerosis. N Engl J Med. 2010 Feb;362(5):387-401.

24 Polman $\mathrm{CH}$, O'Connor PW, Havrdova E, Hutchinson M, Kappos L, Miller DH, et al.; AFFIRM Investigators. A randomized, placebo-controlled trial of natalizumab for relapsing multiple sclerosis. N Engl J Med. 2006 Mar;354(9):899-910.

25 Clifford DB, De Luca A, Simpson DM, Arendt G, Giovannoni G, Nath A. Natalizumab-associated progressive multifocal leukoencephalopathy in patients with multiple sclerosis: lessons from 28 cases. Lancet Neurol. 2010 Apr;9(4):438-46.

26 Bloomgren G, Richman S, Hotermans C, Subramanyam M, Goelz S, Natarajan A, et al. Risk of natalizumab-associated progressive multifocal leukoencephalopathy. N Engl J Med. 2012 May;366(20):1870-80.

27 Frisell T, Forsberg L, Nordin N, Kiesel C, Alfredsson L, Askling J, et al. Comparative analysis of first-year fingolimod and natalizumab drug discontinuation among Swedish patients with multiple sclerosis. Mult Scler. 2016 Jan;22(1):85-93.

28 Izquierdo G, Damas F, Páramo MD, Ruiz-Peña JL, Navarro G. The real-world effectiveness and safety of fingolimod in relapsing-remitting multiple sclerosis patients: an observational study. PLoS One. 2017 Apr;12(4): e0176174. 